\title{
INSULIN RESISTANCE AND ENDOTHELIAL DYSFUNCTION IN RHEUMATOID ARTHRITIS
}

\author{
Alexandru Caraba ${ }^{1}$, Viorica Crisan ${ }^{2}$, Ioan Romosan ${ }^{1}$, Marius Murariu $^{1}$ \\ ${ }^{1}$ Victor Babes, University of Medicine and Pharmacy, Timisoara \\ ${ }^{2}$ Rheumatology Department, City Hospital, Timisoara
}

\begin{abstract}
Rheumatoid arthritis is a chronic inflammatory disease, in which, besides articular involvement, cardiovascular complications due to premature and accelerated atherosclerosis represent a serious problem. Endothelial dysfunction is the first step in the atherosclerosis development. Rheumatoid arthritis associated inflammation contributes to endothelial dysfunction through direct action of cytokines on endothelium, or via increased insulin resistance. The aim of this study was represented by the assessment of endothelial dysfunction in rheumatoid arthritis patients, and correlation of this with impaired insulin sensitivity.

Material and methods. The study was performed on a group of 35 patients with newly diagnosed rheumatoid arthritis. In all patients were assessed: rheumatoid arthritis activity (DAS28), insulin resistance (HOMA-IR) and endothelial dysfunction by means of flow-mediated dilation (FMD).

Results. Inflammation was present in all patients, and the disease activity was high (DAS28: 6.41 \pm 0.94 ). The studied patients showed high insulin resistance (HOMA-IR $5.97 \pm 1.89$ ) and endothelial dysfunction (FMD $7.94 \pm$ $0.91 \%)$. There were significant correlations between endothelial dysfunction and insulin resistance $(p=0.0174)$, respective disease activity $(p=0.0201)$. On the other hand, it was shown a correlation between insulin resistance and the disease activity $(p=0.0290)$.

Conclusion. Endothelial dysfunction was present even in newly diagnosed rheumatoid arthritis patients, contributing to the development of later atherosclerotic cardiovascular disease. Insulin resistance represented an important factor involved in endothelial dysfunction appearance.
\end{abstract}

Keywords: endothelial dysfunction, insulin resistance, rheumatoid arthritis

Rheumatoid arthritis (RA) is a chronic inflammatory disease, in which, besides articular involvement, extraarticular manifestations contribute to increased morbidity and mortality associated with this. Among these extraarticular manifestations, cardiovascular complications due to premature and accelerated atherosclerosis represent a serious problem of these patients $(1,2)$. Lindhardsen et al. demonstrated that the risk of myocardial infarction in RA patients is the same as in type 2 diabetes mellitus (3). Endothelial dysfunction represents the key event in the atherosclerosis development (4).

The traditional risk factors of atherosclerosis, including arterial hypertension, dyslipidemia, diabetes mellitus, smoking and sedentary life style, couldn't explain the great extension of this process, seen in rheumatoid patients (5). In recent years, research conducted revealed that RA-related inflammation is responsible not only for synovial lesions, but it is involved in the development of premature, accelerated atherosclerosis, with subsequent increased risk of atherosclerotic cardiovascular disease $(6,7)$. Inflammation contributes to endothelial dysfunction through direct action of cytokines on endothelium, or via increased insulin resistance (8).

At the moment of diagnosis and during the RA flares, the levels of pro-inflammatory cytokines, including tumour necrosis factor (TNF)- $\alpha$ and interleukin-6 (IL-6) are greatly elevated. Rho et al. demonstrated that the elevated levels of TNF- $\alpha$ and IL- 6 are associated with RA-related atherosclerosis, independent of Framingham risk score (7). TNF- $\alpha$ induces insulin resistance, which is associated with atherosclerosis (7). In present, it is known that antiTNF- $\alpha$ therapy reduces the cardiovascular risk in patients with RA (9). In their study, GonzalezJuanatey et al. demonstrated an improvement of endothelial function following the anti-TNF- $\alpha$ in RA

Correspondence address:

Alexandru Caraba, MD, Department of Internal Medicine, Victor Babes University of Medicine and Pharmacy, 2 Eftimie Murgu Square, Timisoara, Romania

E-mail: alexcaraba@yahoo.com 
patients (10). Improvement of endothelial function is associated with a decrease of inflammation, and restoration of insulin sensitivity $(11,12)$. IL-6 contributes to insulin resistance in patients with RA, and to development of endothelial dysfunction, too $(13,14)$.

The aim of this study is represented by the assessment of endothelial dysfunction in RA patients, and correlation of this with impaired insulin sensitivity, and levels of TNF- $\alpha$ and IL-6.

\section{MATERIAL AND METHODS}

\section{Patients}

The study was performed on a group of 35 patients with newly diagnosed RA. The diagnosis of RA was established based on 2010 American College of Rheumatology/European League Against Rheumatism Classification Criteria for Rheumatoid Arthritis (15). Exclusion criteria were: previous steroid therapy or drugs that alter insulin sensitivity, diabetes mellitus, uncontrolled arterial hypertension, dyslipidemia, chronic kidney disease. All the patients gave their informed consent.

\section{Methods}

In all patients were determined: anti-citrullinated peptide antibodies (Chemiluminescent Microparticle Immunoassay, serum), rheumatoid factor (turbidimetry, serum), erythrocyte sedimentation rate (ESR) (Electro Optical System Technologies), C-reactive protein (turbidimetry, serum), fibrinogen (coagulation, plasma citrate), TNF- $\alpha$ (chemiluminescence immunoassay, serum), IL-6 (electrochemiluminescence immunoassay, serum), fasting insulinemia (chemiluminescence immunoassay, serum) and glycemia (photometry, plasma NaF K2 oxalate).

Rheumatoid arthritis activity was assessed using DAS28. DAS28 was calculated based on: ESR, tender joint count (28 joints), swollen joint count (28 joints), and the patient's assessment of global wellbeing (100 $\mathrm{mm}$ visual analogue scale). (http:// www.4s-dawn.com/DAS28/DAS28.html)

Insulin resistance was assessed by Homeostasis Model Assessment of Insulin Resistance (HOMAIR) index, using fasting insulin and glucose (16).

Endothelial dysfunction was assessed by means of flow mediated vasodilation, on brachial artery, using B-mode ultrasonography (Siemens Acuson X300
Ultrasound System, with linear transducer of 10 $\mathrm{MHz}$ ). Before the test, the patient was relaxed at a stable room temperature between $20-25^{\circ} \mathrm{C}$; smoking, ingestion of caffeine, high-fat foods and vitamin $\mathrm{C}$ were prohibited. The diameter of the brachial artery was measured incident with the $\mathrm{R}$ wave of the electrocardiograph trace (Di). Then, ischemia was induced by inflating the pneumatic cuff to a pressure $50 \mathrm{mmHg}$ above systolic one, in order to obliterate the brachial artery and induce ischaemia. After 5 minutes, the cuff was deflated and the diameter was measured after 60 seconds post-deflation (Df). FMD was calculated with the formula: $F M D=[(D f-D i) /$ Di] $\times 100(17)$.

\section{Statistical analysis}

Data were expressed as mean \pm standard deviation. Correlations were investigated using Pearson's test. $p$ values of less than 0.05 were considered statistically significant.

\section{RESULTS}

The group of patients with RA was formed by 12 males and 23 females, with the mean age of $55.6 \pm$ 9.74 years. All the patients were positive for rheumatoid factor and anti-citrullinated peptide antibodies.

The demographic data and laboratory findings are presented in the Table 1 .

TABLE 1. Demographic data and laboratory findings in RA patients

\begin{tabular}{|l|l|}
\hline Parameter & Value (mean \pm standard deviation) \\
\hline Males/Females & $12 / 23$ \\
\hline Mean age (years) & $55.6 \pm 9.74$ \\
\hline ESR $(\mathrm{mm} / \mathrm{h})$ & $74.11 \pm 18.47$ \\
\hline C-reactiv protein $(\mathrm{mg} / \mathrm{l})$ & $60.34 \pm 27.8$ \\
\hline Fibrinogen $(\mathrm{mg} / \mathrm{dl})$ & $693.52 \pm 284.61$ \\
\hline DAS28 & $6.41 \pm 0.94$ \\
\hline TNF $\alpha(\mathrm{pg} / \mathrm{ml})$ & $89.65 \pm 21.41$ \\
\hline IL6 $(\mathrm{pg} / \mathrm{ml})$ & $89.40 \pm 20.08$ \\
\hline FMD $(\%)$ & $7.94 \pm 0.81$ \\
\hline Fasting insulin $(\mathrm{mlU} / \mathrm{l})$ & $25.88 \pm 6.28$ \\
\hline Fasting glucose $(\mathrm{mg} / \mathrm{dl})$ & $89.94 \pm 11.57$ \\
\hline HOMA-IR & $5.97 \pm 1.89$ \\
\hline
\end{tabular}

Inflammation was present in all patients, and the disease activity was high (DAS28: $6.41 \pm 0.94)$. The studied RA patients showed high insulin resistance (HOMA-IR $5.97 \pm 1.89$ ) and endothelial dysfunction (FMD $7.94 \pm 0.91 \%$ ). The correlations between endothelial dysfunction and insulin resistance, respective disease activity are presented in Table 2. 
TABLE 2. Correlations between endothelial dysfunction and insulin resistance, respective disease activity

\begin{tabular}{|l|c|c|}
\hline Correlation & $\mathbf{r}$ & $\mathbf{p}$ \\
\hline FMD-HOMA-IR & -0.39958 & 0.0174 \\
\hline FMD-DAS28 & -0.39123 & 0.0201 \\
\hline FMD-TNF- $\alpha$ & -0.3309 & 0.0345 \\
\hline FMD-IL-6 & -0.34096 & 0.0225 \\
\hline
\end{tabular}

The correlations between insulin resistance and disease activity are shown in Table 3 .

TABLE 3. Correlations between insulin resistance and disease activity

\begin{tabular}{|l|c|c|}
\hline Correlation & $\mathbf{r}$ & $\mathbf{p}$ \\
\hline HOMA-IR-DAS28 & 0.369224 & 0.0290 \\
\hline HOMA-IR-TNF- $\alpha$ & 0.353472 & 0.0372 \\
\hline HOMA-IR-IL-6 & 0.341212 & 0.0448 \\
\hline
\end{tabular}

The results show that at the moment of RA diagnosis, there are significant correlations between endothelial dysfunction and insulin resistance (Fig. 1), respective disease activity, expressed as DAS28 (Fig. 2). On the other hand, insulin resistance is correlated with the disease activity (Fig. 3) and the levels of pro-inflammatory cytokines (TNF- $\alpha$, IL-6).

\section{DISCUSSION}

RA is a chronic, systemic inflammatory disorder of unknown etiology, associated with a high risk for cardiovascular diseases, due to accelerated and premature atherosclerosis (16). In RA patients, the inci-
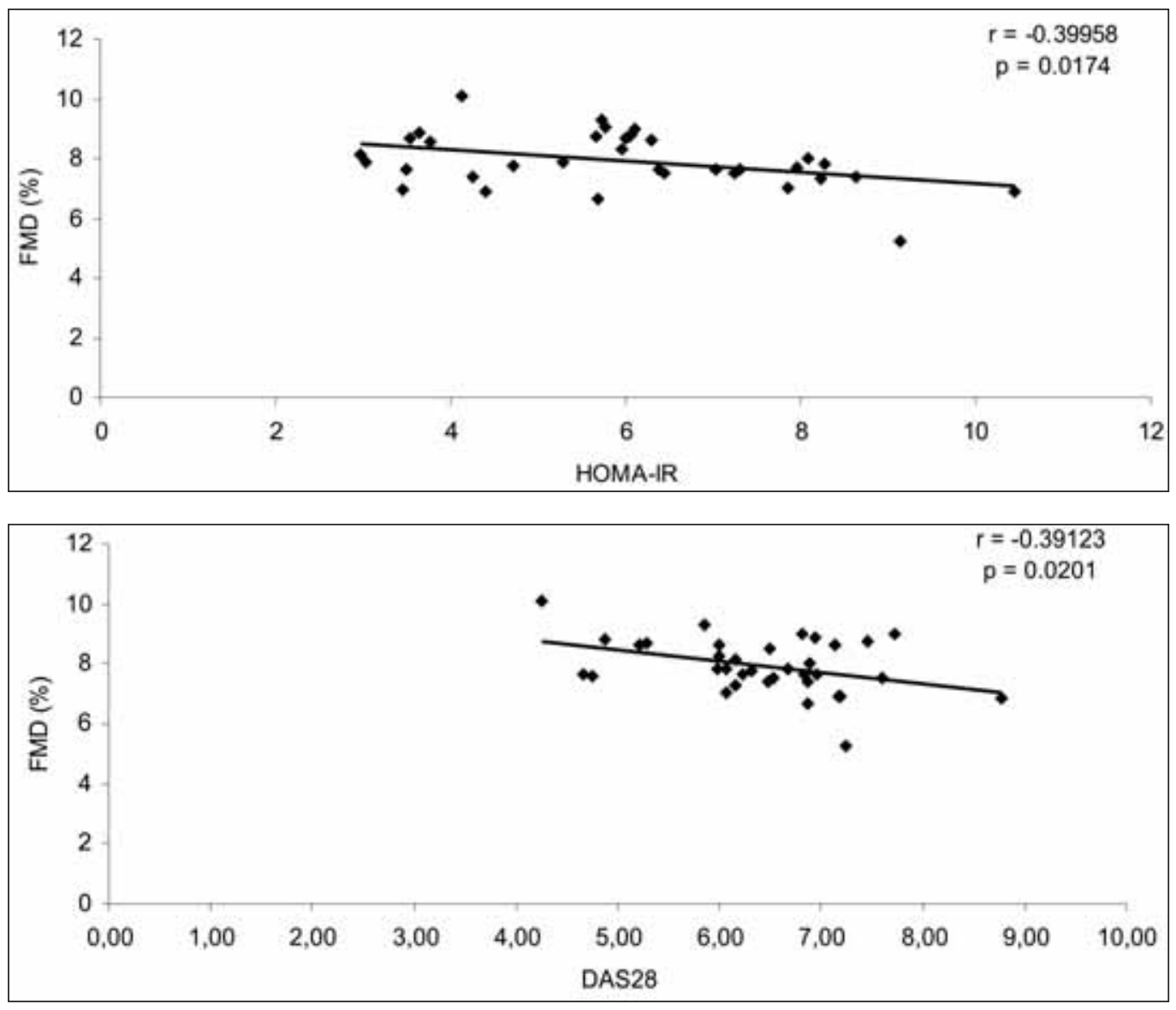

FIGURE 1.

Correlation between endothelial dysfunction and insulin resistance
FIGURE 2.

Correlation between endothelial dysfunction and RA activity

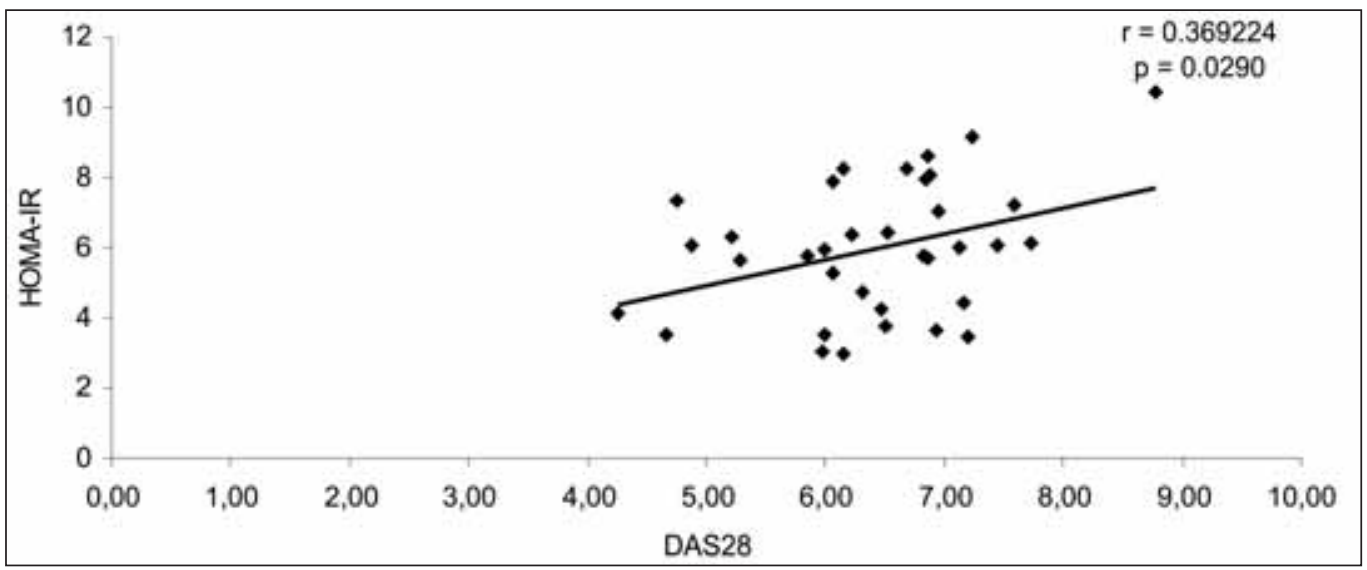

FIGURE 3.

Correlation between insulin resistance and $R A$ activity 
dence of cardiovascular events is more than three times than in general population. Atherosclerotic cardiovascular disease represents the leading cause of death in these patients (18). Accumulating evidence showed that the traditional risk factors of atherosclerosis can not explain the magnitude of this process in these patients $(5,18)$. But systemic inflammation associated with RA represents the key, which can explain the premature development of atherosclerosis. Inflammation is present in all stages of atherosclerosis development, from endothelial dysfunction to plaque rupture (18).

Pro-inflammatory cytokines as TNF- $\alpha$ and IL-6 are involved in endothelial dysfunction appearance, acting on the vascular endothelium, or through insulin sensitivity. These cytokines exert deleterious effects on the vascular endothelium, which may generate the reduction of nitric oxide synthesis and promote endothelial dysfunction. On the other hand, these pro-inflammatory cytokines are involved in insulin resistance appearance, risk factor for endothelial dysfunction (19).

In the present study, it was evaluated the endothelial dysfunction in RA patients and its correlation with insulin resistance. All the studied patients presented impaired flow mediated vasodilation, which was correlated in a negative manner with the insulin resistance $(\mathrm{r}=-0.39958, \mathrm{p}=0.0174)$, as well as in the study performed by Cortes-Rodriguez et al (20).

The presented study showed that the endothelial dysfunction was correlated with the RA activity, expressed as DAS28 $(r=-0.39123, p=0.0201)$. Fernandez-Real revealed that chronic inflammation is linked to endothelial dysfunction (8). Another studies found the same correlation. Studying middle aged RA patients without cardiovascular risk factors or overt cardiovascular diseases, Vaudo et al. identified impaired endothelial reactivity, related with chronic inflammation $(r=-0.47, p<0.05)(21)$. The study performed by Watanabe et al. demonstrated that flow-mediated dilation was inversely correlated with the RA activity $(r=-0.404, p<0.05)(22)$. In Sarli et al. study, it was evidentiated the correlation between flow mediated dilation and disease activity $(p=0.038)(23)$. Klimek et al. identified the existence of the relationship between the severity of in- flammatory response and endothelial dysfunction in RA with short duration of evolution (24). Studying newly diagnosed patients with RA, de Groot et al. showed that the impaired endothelial function was present from the beginning, inversely correlated with the disease activity $(r=-0.31, p=0.016)$. The authors highlighted the role of RA treatment in order to prevent the development of the atherosclerotic cardiovascular disease associated with RA (25).

The present study documented that the impaired insulin sensitivity was correlated with rheumatoid arthritis activity $(r=0.369224, p=0.0290)$, and with serum levels of serum pro-inflammatory cytokines (TNF- $\alpha: r=0.353472, p=0.0372$; IL-6: $r=0.341212$, $\mathrm{p}=0.0448$ ). Dessein and Joffe showed in their study that in RA patients, insulin resistance, expressed as HOMA-IR, was correlated with markers for inflammation and disease activity $(\mathrm{r}=0.208, \mathrm{p}=0.04)$ (26). In another study, performed by La Montagna et al. it was evidentiated the existence of insulin resistance in RA patients and its significant correlation with the disease activity $(r=0.549, p<0.0001)$ (27). Chung et al. emphasized the significant correlations between insulin resistance and RA activity, respective the levels of pro-inflammatory cytokines (TNF- $\alpha$ and IL-6) $(p<0.05)(14)$. In early, untreated RA patients, Shahin et al. documented severe insulin resistance, which was correlated with the disease activity (28). El Sayed et al. showed a significant positive correlations between HOMA-IR levels and disease activity $(r=0.969, p=0.000)(29)$. In another study, it was demonstrated a positive correlation between insulin resistance and DAS28 $(r=0.361, \mathrm{p}<$ 0.005 ), as well as levels of cytokines (TNF- $\alpha$ : $r=$ $0.300, p<0.05$, IL-6: $r=0.329, p<0.05)(2)$.

\section{CONCLUSION}

Endothelial dysfunction is present even in newly diagnosed RA, contributing to the development of later atherosclerotic cardiovascular disease. Insulin resistance, driven by the inflammatory mediators, represents an important factor involved in endothelial dysfunction appearance. By suppressing inflammation, RA treatment contributes to the prevention of cardiovascular disease appearance. 


\section{REFERENCES}

1. Firestein G.S. Evolving concepts of rheumatoid arthritis. Nature 2003; 423: 356-61

2. Chen D.Y., Chen Y.M., Hsieh T.Y. et al. Significat effects of biological therapy on lipid profiles and insulin resistance in patients with rheumatoid arthritis. Arthritis Res Ther 2015; 17: 52

3. Lindhardsen J., Ahlehoff O., Gislason G.H. et al. The risk of myocardial infarction in rheumatoid arthritis and diabetes mellitus: a Danish nationwide cohort study. Ann Rheum Dis 2011; 70: 929-34.

4. Sitia S., Tomasoni L., Atzeni F. et al. From endothelial dysfunction to atherosclerosis. Autoimmunity Reviews 2010; 9(12): 830-834

5. D'Agostino R.B., Vasan R.S., Pencina M.J. et al. General cardiovascular risk profile for use in primary care: the Framingham Heart Study. Circulation 2008; 117: 743-53

6. Sattar N., McCarey D.W., Capell H., McInnes I.B. Explaining how "highgrade" systemic inflammation accelerates vascular risk in rheumatoid arthritis. Circulation 2003; 108: 2957-63

7. Rho Y.H., Chung C.P., Oeser A. et al. Inflammatory mediators and premature coronary atherosclerosis in rheumatoid arthritis. Arthritis Rheum 2009; 61:1580-5

8. Fernandez-Real J.M. Insulin resistance and atherosclerosis. The impact of oxidative stress on endothelial function. Rev Esp Cardiol Sup/ 2008; 8: 42C-49C

9. Westlake S.L., Colebatch A.N., Baird J. et al. Tumour necrosis factor antagonists and the risk of cardiovascular disease in patients with rheumatoid arthritis: a systematic literature review. Rheumatology (Oxford) 2011; 50: 518-31

10. Gonzalez-Juanatey C., Vazquez-Rodriguez T.R., Miranda-Filloy J.A. et al. Anti-TNF-alpha-Adalimumab therapy is associated with persistent improvement of endothelial function without progression of carotid intima-media wall thickness in patients with rheumatoid arthritis refractory to conventional therapy. Mediators of Inflammation 2012, Article ID 674265, http://dx.doi. org/10.1155/2012/674265

11. Gonzalez-Juanatey C., Llorca J., Sanchez-Andrade A. et al. Short-term adalimumab therapy improves endothelial function in patients with rheumatoid arthritis refractory to infliximab. Clin Exp Rheumatol 2006; 24: 309-12

12. Gonzalez-Gay M.A., Gonzalez-Juanatey C., Vazquez-Rodriguez T.R. et al. Insulin resistance in rheumatoid arthritis: the impact of the anti-TNF- $\alpha$ therapy. Ann N Y Acad Sci 2010; 1193: 153-9

13. Abeywardena M.Y., Leifert W.R., Warnes K.E. et al. Cardiovascular biology of interleukin-6. Curr Pharm Des 2009; 15 1809-21

14. Chung C.P., Oeser A., Solus J.F. et al. Inflammation-associated insulin resistance: differential effects in rheumatoid arthritis and systemic lupus erythematosus define potential mechanisms. Arthritis Rheum 2008; 58: 2105-12

15. Aletaha D., Neogi T., Silman A.J. et al. The 2010 American College of Rheumatology/European League Against Rheumatism classification criteria for rheumatoid arthritis. Ann Rheum Dis 2010; 69: $1580-8$

16. Ferraz-Amaro I., González-Juanatey C., López-Mejias R. et al. Metabolic Syndrome in Rheumatoid Arthritis. Mediators of Inflammation, 2013, Article ID 710928, doi:10.1155/2013/710928

17. Corretti M.C., Anderson T.J., Benjamin E.J. et al. Guidelines for the ultrasound assessment of endothelial-dependent flow-mediated vasodilation of the brachial artery: a report of the International Brachial Artery Reactivity Task Force. J Am Coll Cardiol 2002; 39(2): 257-65

18. Choy E. Understanding the dynamics: pathways involved in the pathogenesis of rheumatoid arthritis. Rheumatology 2012; 51(suppl 5): v3-v11

19. Sandoo A., van Zanten J.C.S., Metsios G.S., et al. Vascular function and morphology in rheumatoid arthritis: a systematic review. Rheumatology (Oxford) 2011; 50(11): 2125-2139

20. Cortes-Rodriguez B., Fernandez de la Puebla Gimenez R.A., Morote Ibarrola G. et al. Endothelial dysfunction is associated with insulin resistance in patients with rheumatoid arthritis. Med Clin (Barc) 2007; 128(1): 414-6

21. Vaudo G., Marchesi S., Gerli R. et al. Endothelial dysfunction in young patients with rheumatoid arthritis and low disease activity. Ann Rheum Dis 2004; 63(1): 31-5

22. Watanabe T., Takemura M., Sato M. et al. Clinical significance of brachial flow-mediated dilation in patients with rheumatoid arthritis. Int J Rheum Dis 2014; 17(1): 26-33

23. Sarli B., Baktir A.O., Cebicci M., et al. Predictors of endothelial dysfunction in patients with rheumatoid arthritis. Angiology 2014; 65(9): 778-82

24. Klimek E., Skalska A., Kwaśny-Krochin B., et al. Differential associations of inflammatory and endothelial biomarkers with disease activity in rheumatoid arthritis of short duration. Mediators of Inflammation 2014, Article ID 681635, doi:10.1155/2014/681635

25. de Groot L., Jager N.A., Westra J., et al. Does reduction of disease activity improve early markers of cardiovascular disease in newly diagnosed rheumatoid arthritis patients? Rheumatology (Oxford) 2015; 54(7): 1257-61

26. Dessein P.H., Joffe B.I. Insulin resistance and impaired beta cell function in rheumatoid arthritis. Arthritis Rheum 2006; 54: 2765-75

27. La Montagna G., Cacciapuoti F., Buono R., et al. Insulin resistance is an independent risk factor for atherosclerosis in rheumatoid arthritis. Diabets Vasc Dis Res 2007; 4: 130-5

28. Shahin D., Eltoraby E., Mesbah A., Houssen M. Insulin resistance in early untreated rheumatoid arthritis patients. Clin Biochem 2010; 43(7-8): 661-5

29. El Sayed A.M., El Bakry S.A., Mobasher S.A., et al. Insulin resistance as a risk factor for subclinical atherosclerosis in rheumatoid arthritis. The Egyptian Rheumatologist 2014; 36(1): 3-7 